\title{
Kanamycin Sulfate
}

National Cancer Institute

\section{Source}

National Cancer Institute. Kanamycin Sulfate. NCI Thesaurus. Code C65998.

The sulfate salt of kanamycin, a naturally occurring aminoglycoside antibiotic with antimicrobial property. Derived from Streptomyces kanamyceticus, kanamycin is an antibiotic complex consisting of three components: kanamycin A, the major component (also usually designated as kanamycin), and kanamycins B and C, the minor components. This agent irreversibly binds to the bacterial 30S ribosomal subunit. Specifically, this antibiotic is lodged between 16S rRNA and S12 protein within the 30S subunit. This leads to interference with translational initiation complex, misreading of mRNA, thereby hampering protein synthesis and resulting in bactericidal effect. Aminoglycosides are mostly ineffective against anaerobic bacteria, fungi and viruses. 